\title{
Reactivation of VaSTS1 expression in Arabidopsis thaliana transgenic plants by retransformation with $2 b$ from the Cucumber Mosaic Virus isolate NK
}

\author{
N.N. Nityagovsky ${ }^{1,2 *}$, A.P. Tyunin ${ }^{1}$, K.V. Kiselev ${ }^{1,2}$ \\ 'Laboratory of Biotechnology, Federal Scientific Center of East Asia Terrestrial Biodiversity, Far Eastern Branch of Russian Academy of Sciences, \\ Vladivostok, Russia \\ ${ }^{2}$ Department of Biochemistry and Biotechnology, Far Eastern Federal University, Vladivostok, Russia
}

DOI 10.18699/ICG-PlantGen2019-45

(c) Autors, 2019

* e-mail: niknit1996@gmail.com

\begin{abstract}
Since transgene silencing in genetically transformed plants is a serious limitation for a wide application of genetic engineering techniques, studying mechanisms ensuring stability of transgene expression is vital. Multiple investigations concerning viral suppressors of gene silencing revealed that protein $2 \mathrm{~b}$ from Cucumovirus (CMV) effectively represses the RNA-induced silencing complex. The current study presents unique data on using the $2 \mathrm{~b}$ gene from the CMV isolate NK for a transgene silencing reduction in A. thaliana plants earlier transformed with VaSTS1 from Vitis amurensis Rupr. In our study, two VaSTS1 transgenic lines with decreased expression of VaSTS1 increased transgene expression up to 3.0-fold upon retransformation with $2 \mathrm{~b}$ from CMV NK. Thus, $2 \mathrm{~b}$ from CMV NK can reactivate a silenced transgene.

Key words: protein 2b; Cucumovirus; stilbenes; stilbene synthase; $t$-resveratrol; transgene silencing.
\end{abstract}

\section{Introduction}

Agrobacterium-mediated transformation is a common method to generate transgenic plants. However, transgene silencing is a serious restriction occurring in plants and plant cell cultures, frequently correlating with a high transgene copy number, the use of strong promoters, insertion locations of transgenes, occurring during integration (Butaye et al., 2005). Studies concerning the mechanisms leading to transgene silencing revealed that RNA interference is a key regulator of both endogenous and introduced gene sequences at transcriptional gene silencing (TGS) and post-transcriptional gene silencing levels (PTGS; Law, Jacobsen, 2010). This complex system involving short non-coding RNA to suppress exogenous sequences seems to be a result of a long co-evolution of plants and phytoviruses (Pumplin, Voinnet, 2013). Among the other phytoviruses, Cucumber mosaic virus (CMV) in the genus Cucumovirus of the family Bromoviridae has the broadest spectrum of known plant hosts, including more than 1200 species of herbaceous plants, shrubs, and trees referring to more than 100 different families (Rossinck, 2002). Multiple studies concerning the virulence of CMV strains assigned protein $2 \mathrm{~b}$ to be the key factor responsible for induction of symptoms, systemic necrosis, and synergic symptom induction by other viruses in the case of co-infection (Rossinck, 2002). Moreover, protein $2 \mathrm{~b}$ was shown to counter basal mechanisms of plant pathogen defense, including RNA silencing (Lewsey et al., 2010). Possessing two nuclear localization signals, CMV protein $2 \mathrm{~b}$ was shown to bind with double-stranded RNAs in the nucleus, which is required for a proper RNA-induced silencing complex (RISC) assembly and targeting. Moreover, $2 b$ of the CMV isolate Fny is able to block the PAZ domain of Argonaut 1 (AGO1), a RNA-slicing protein, a critical part of the plant RISC complex (Zhang et al., 2006).
Taking into consideration the fact that the suppression of transgenes introduced into plant genomes is associated with RNA silencing, the further development of genetic transformation techniques will overall benefit from the use of viral suppressors of silencing. Our recent study conducted on the rolB-transgenic cell culture VB2 of the wild-growing grape Vitis amurensis Rupr., which showed a significant reduction in $r o l B$ expression, revealed that transgene expression can be restored by retransformation with $2 b$ from the CMV isolate NK even after 10 years of continuous subcultivation (Dubrovina, Kiselev, 2012; Tyunin et al., 2019). Furthermore, retransformation with $2 b$ from CMV NK significantly enhanced the production level of pharmaceutically-valuable $t$-resveratrol in $2 b$-retransformed callus cell line VB2 by induction of stilbene synthase (VaSTS) genes encoding $t$-resveratrol biosynthesis enzymes.

In order to find an alternative biological model for the production of valuable $t$-resveratrol, Arabidopsis thaliana plants were transformed with the VaSTS1 gene responsible for $t$-resveratrol biosynthesis in $V$. amurensis (Tyunin et al., 2018). The derived homozygous $A$. thaliana transgenic lines, ST1-1 and ST1-3, demonstrated different levels of VaSTS1 transgene expression after several generations, suggesting transgene sequence silencing due to the RNAi mechanism. In the course of the current study, both ST1-1 and ST1-3 plants were retransformed with $2 b$ from CMV NK, and homozygous transformants were selected and analyzed for VaSTS1 transgene expression and the cytosine DNA methylation level within the VaSTS1 nucleotide sequence. Our data show a strong negative correlation between the transgene expression and the level of cytosine methylation within the VaSTS1 sequence. Furthermore, our data show the reactivation of VaSTS1 expression in response to retransformation with $2 b$ 
Table 1

$2 b$ and VaSTS1 expression and the average level of cytosine methylation of the VaSTS1 sequence in the $A$. thaliana lines

\begin{tabular}{llll}
\hline Line name & 2b expression, r.u. & VaSTS1 expression, r.u. & $\begin{array}{l}\text { Average level of cytosine methylation } \\
\text { within 3'-end of VaSTS1 sequence, \% }\end{array}$ \\
\hline KA-0 & $\mathrm{n} / \mathrm{a}$ & $\mathrm{n} / \mathrm{a}$ & $\mathrm{n} / \mathrm{a}$ \\
KA-0-2b-Ib-5p & $0.46 \pm 0.05$ & $\mathrm{n} / \mathrm{a}$ & $\mathrm{n} / \mathrm{a}$ \\
ST1-1 & $\mathrm{n} / \mathrm{a}$ & $0.43 \pm 0.06$ & 17.79 \\
ST1-1-2b-Ic-1p & $0.62 \pm 0.07$ & $0.20 \pm 0.04$ & 25.24 \\
ST1-1-2b-Ila-2p & $0.49 \pm 0.06$ & $0.53 \pm 0.05$ & 5.24 \\
ST1-1-2b-IId-2p & $0.46 \pm 0.03$ & $0.31 \pm 0.05$ & 2.44 \\
ST1-3 & $\mathrm{n} / \mathrm{a}$ & $0.13 \pm 0.04$ & 13.42 \\
ST1-3-2b-Ila-3p & $0.32 \pm 0.04$ & $0.11 \pm 0.02$ & 16.65 \\
ST1-3-2b-IIb-3p & $0.72 \pm 0.02$ & $0.36 \pm 0.08$ & 1.02 \\
ST1-3-2b-Ilb-5p & $0.77 \pm 0.03$ & $0.13 \pm 0.02$ & 29.38 \\
\hline
\end{tabular}

from CMV due to a decrease in the level of VaSTS1 cytosine methylation.

\section{Materials and methods}

A. thaliana transgenic lines ST1-1 and ST1-3 were obtained using the method of dipping inflorescences into the agrobacteria suspension as described previously (Dubrovina et al., 2017). Binary vector constructs contained the gene of interest and an antibiotic resistance gene on the double $35 \mathrm{~s}$ CaMV promoter.

A. thaliana transgenic lines KA-0 and VaSTS1 were transformed in the current study with Agrobacterium tumefaciens strain GV3101::pMP90 containing binary vector construct pZP-RCS2-2b-hpt. The selection of the obtained $2 b$ trangenic lines was performed on W0 media with kanamycin and hygromycin.

Transgenic lines KA-0, ST1-1 and ST1-3 were obtained by inoculation of wild-type $A$. thaliana (ecotype: Columbia) with $A$. tumefaciens strains GV3101::pMP90 containing binary vector constructs pZP-RCS2-nptII (empty vector) and pZP-RCS2-VaSTS1-nptII, respectively.

The $A$. thaliana transgenic plants were grown in planting pots using a commercially available universal potting mix in a climatic chamber (Panasonic MLR-352, Japan) at $22{ }^{\circ} \mathrm{C}$ under day $(16 \mathrm{~h})$ /night $(8 \mathrm{~h})$ conditions at an illumination of $\sim 120 \mu \mathrm{moL} \mathrm{m}^{-2} \mathrm{~s}^{-1}$.

DNA and RNA were extracted from the $2 b$ transgenic lines and cDNA was obtained from RNA as previously described (Tyunin et al., 2019). Expression analysis of the VaSTS1 and $2 b$ genes in $A$. thaliana was performed using real-time PCR (RT-PCR) according to the SYBR Green method.

Data on the level of cytosine methylation of the nucleotide sequence in the VaSTS1 transgenic lines were obtained by bisulfite sequencing as described previously (Kiselev et al., 2015).

\section{Results and discussion}

In the course of our study, A. thaliana transgenic plants ST1-1 and ST1-3 overexpressing VaSTS1 from $V$. amurensis were tested for the transgene expression level by qRT-PCR and compared to the control line KA-0 transformed with an empty vector (Table 1).

The data demonstrated a high level of VaSTS1 expression for ST1-1 plants, while in ST1-3 plants it was one-third as high. In order to test if retransformation with $2 b$ from CMV is able to restore VaSTS1 expression in ST1-3 transgenic plants, all lines mentioned were retransformed, and homozygous A. thaliana plants were analyzed (Table 1).

According to the data obtained, retransformation of ST1-1 plants had no significant effect on VaSTS1 expression, with the exception of line ST1-1-2b-Ic-1p, which showed a nonsignificant decrease in the level of VaSTS1 expression. However, retransformation of ST1-3 plants led to a strong increase in VaSTS1 expression in line ST1-3-2b-IIb-3p, reaching a level comparable with that of the high-expression line ST1-1.

According to the data on the level of cytosine methylation within the 3' end of the VaSTS1 transgene presented in Table 1 , both initial lines show comparable levels of methylated cytosines from 17.7 to $13.4 \%$ of the total amount of cytosine residues.

However, strong induction of VaSTS1 expression in ST1$3-2 b-I I b-3 p$ plants after retransformation with $2 b$ seems to be the result of the demethylation process within the 3 ' end of the transgene sequence, as the total level of methylated cytosines was reduced to $1.0 \%$.

\section{Conclusions}

Our data show that retransformation with $2 b$ from CMV can reactivate expression of a silenced transgene in model Arabidopsis thaliana plants. Reactivation of transgene expression is strongly connected to demethylation of cytosine residues within the nucleotide sequences of a reactivating transgene.

\section{References}

Butaye K.M.J., Cammue B.P.A., Delaure S.L., De Bolle M.F.C. Approaches to minimize variation of transgene expression in plants. Mol. Breed. 2005;16:79-91. DOI 10.1007/s11032-005-4929-9.

Dubrovina A.S., Kiselev K.V. Effect of long-term cultivation on resveratrol accumulation in a high-producing cell culture of Vitis amurensis. Acta Physiol. Plant. 2012;34:1101-1106. DOI 10.1007/s11738011-0907-5. 
Dubrovina A.S., Kiselev K.V., Khristenko V.S., Aleynova O.A. The calcium-dependent protein kinase gene $\mathrm{VaCPK} 29$ is involved in grapevine responses to heat and osmotic stresses. Plant Growth Regul. 2017;82:79-89. DOI 10.1007/s10725-016-0240-5.

Kiselev K.V., Tyunin A.P., Ogneva Z.V., Dubrovina A.S. Age-associated alterations in the somatic mutation level in Arabidopsis thaliana. Plant Growth Regul. 2015;75:493-501. DOI 10.1007/s10725-0140012-z.

Law J.A., Jacobsen S.E. Establishing, maintaining and modifying DNA methylation patterns in plants and animals. Nat. Rev. Genet. 2010; 11:204-220. DOI 10.1038/nrg2719.

Lewsey M.G., Murphy A.M., MacLean D., Dalchau N., Westwood J.H., Macaulay K., Bennett M.H., Moulin M., Hanke D.E., Powell G., Smith A.G., Carr J.P. Disruption of two defensive signaling pathways by a viral RNA silencing suppressor. Mol. Plant-Microbe Interact. 2010;23:835-845. DOI 10.1094/MPMI-23-7-0835.

Pumplin N., Voinnet O. RNA silencing suppression by plant pathogens: defence, counter-defence and counter-counter-defence. Nat. Rev. Microbiol. 2013;11:745-760. DOI 10.1038/nrmicro3120.

Roossinck M.J. Evolutionary history of Cucumber mosaic virus deduced by phylogenetic analyses. J. Virol. 2002;76:3382-3387. DOI 10.1128/JVI.76.7.3382-3387.2002.
Tyunin A.P., Nityagovsky N.N., Grigorchuk V.P., Kiselev K.V. Stilbene content and expression of stilbene synthase genes in cell cultures of Vitis amurensis treated with cinnamic and caffeic acids. Biotechnol. Appl. Biochem. 2018;65:150-155. DOI 10.1002/bab.1564.

Tyunin A.P., Nityagovsky N.N., Karetin Y.A., Kiselev K.V. Reactivation of rolB transgene expression in Vitis amurensis Rupr. cells upon retransformation with $2 b$ gene from Cucumovirus isolate NK. Biotechnol. Lett. 2019;41:443-445. DOI 10.1007/s10529-018-02642-6.

Tyunin A.P., Suprun A.R., Nityagovsky N.N., Manyakhin A.Y., Karetin Y.A., Dubrovina A.S., Kiselev K.V. The effect of explant origin and collection season on stilbene biosynthesis in cell cultures of Vitis amurensis Rupr. Plant Cell Tissue Organ Cult. 2019;136:189-196. DOI 10.1007/s11240-018-1490-x.

Zhang X., Yuan Y.R., Pei Y., Lin S.S., Tuschl T., Patel D.J., Chua N.H. Cucumber mosaic virus-encoded $2 \mathrm{~b}$ suppressor inhibits Arabidopsis Argonaute1 cleavage activity to counter plant defense. Genes Dev. 2006;20:3255-3268. DOI 10.1101/gad.1495506.

Acknowledgements. The work is supported by The Russian Foundation for Basic Research, grant number: 17-04-01381.

Conflict of interest. The authors declare no conflict of interest. 